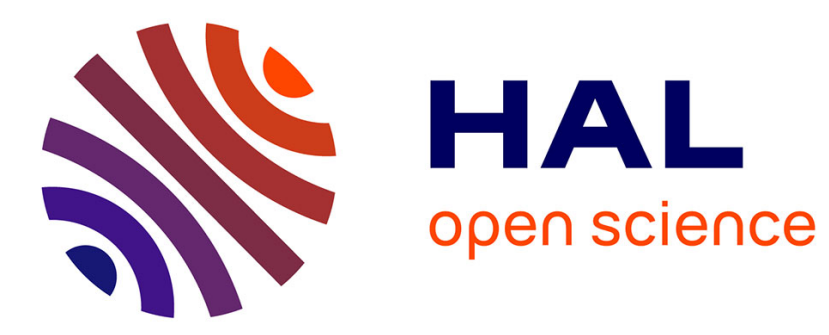

\title{
Indefinite Linear MPC and Approximated Economic MPC for Nonlinear Systems
}

Mario Zanon, Sebastien Gros, Moritz Diehl

\section{To cite this version:}

Mario Zanon, Sebastien Gros, Moritz Diehl. Indefinite Linear MPC and Approximated Economic MPC for Nonlinear Systems. NETCO 2014 - New Trends in Optimal Control, Jun 2014, Tours, France. hal-01030531

\section{HAL Id: hal-01030531 https://hal.inria.fr/hal-01030531}

Submitted on 22 Jul 2014

HAL is a multi-disciplinary open access archive for the deposit and dissemination of scientific research documents, whether they are published or not. The documents may come from teaching and research institutions in France or abroad, or from public or private research centers.
L'archive ouverte pluridisciplinaire HAL, est destinée au dépôt et à la diffusion de documents scientifiques de niveau recherche, publiés ou non, émanant des établissements d'enseignement et de recherche français ou étrangers, des laboratoires publics ou privés. 


\title{
DOPTEC
}

\section{ITN SADCO}

Initial Training Network

Sensitivity Analysis for Deterministic Controller Design

Sensitivity Anatysis for Deterministic Controller Design

\section{Indefinite Linear MPC and Approximated Economic MPC for Nonlinear Systems}

\author{
Mario Zanon ${ }^{1}$, Sébastien Gros ${ }^{2}$, Moritz Diehl ${ }^{1}$
}

1. KU Leuven \& Univ. of Freiburg, 2. Chalmers Univ.

NetCo conference, Tours 2014 


\section{Outline}

(1) Economic MPC and Stability Analysis

(2) The Linear Quadratic Case

(3) Approximated EMPC with stability guarantees 
(1) Economic MPC and Stability Analysis 


\section{Economic MPC and Stability Analysis}

MPC

Optimal Control Problem

$$
\min _{x_{0}, u_{0}, \ldots, x_{N}}
$$

$$
\text { s.t. }
$$




\section{Economic MPC and Stability Analysis}

MPC

Optimal Control Problem

$$
\min _{x_{0}, u_{0}, \ldots, x_{N}}
$$

$$
\text { s.t. } x_{0}-\bar{x}_{i}=0 \text {, }
$$




\section{Economic MPC and Stability Analysis}

MPC

Optimal Control Problem

$$
\min _{x_{0}, u_{0}, \ldots, x_{N}}
$$

$$
\begin{aligned}
& \text { s.t. } x_{0}-\bar{x}_{i}=0 \text {, } \\
& x_{k+1}-f\left(x_{k}, u_{k}\right)=0 \text {, }
\end{aligned}
$$




\section{Economic MPC and Stability Analysis}

MPC

Optimal Control Problem

$$
\begin{array}{cl}
\min _{x_{0}, u_{0}, \ldots, x_{N}} & \\
\text { s.t. } & x_{0}-\bar{x}_{i}=0 \\
& x_{k+1}-f\left(x_{k}, u_{k}\right)=0 \\
& x_{N}-x_{N}^{s}=0
\end{array}
$$

Initial condition

System dynamics

Terminal constraint 


\section{Economic MPC and Stability Analysis}

MPC

Optimal Control Problem

$$
\begin{aligned}
\min _{x_{0}, u_{0}, \ldots, x_{N}} & \sum_{k=0}^{N-1} I\left(x_{k}, u_{k}\right) \\
\text { s.t. } & x_{0}-\bar{x}_{i}=0, \\
& x_{k+1}-f\left(x_{k}, u_{k}\right)=0, \\
& x_{N}-x_{N}^{s}=0 .
\end{aligned}
$$

(Quadratic) stage cost

Initial condition

System dynamics

Terminal constraint 


\section{Economic MPC and Stability Analysis}

MPC

Optimal Control Problem

$$
\begin{aligned}
\min _{x_{0}, u_{0}, \ldots, x_{N}} & \sum_{k=0}^{N-1} I\left(x_{k}, u_{k}\right) \\
\text { s.t. } & x_{0}-\bar{x}_{i}=0, \\
& x_{k+1}-f\left(x_{k}, u_{k}\right)=0, \\
& x_{N}-x_{N}^{s}=0 .
\end{aligned}
$$

(Quadratic) stage cost

Initial condition

System dynamics

Terminal constraint
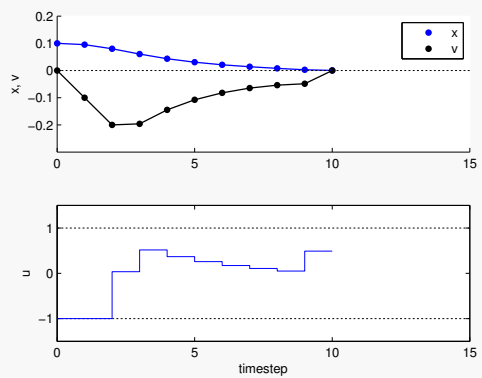


\section{Economic MPC and Stability Analysis}

MPC

Optimal Control Problem

$$
\begin{aligned}
\min _{x_{0}, u_{0}, \ldots, x_{N}} & \sum_{k=0}^{N-1} I\left(x_{k}, u_{k}\right) \\
\text { s.t. } & x_{0}-\bar{x}_{i}=0, \\
& x_{k+1}-f\left(x_{k}, u_{k}\right)=0, \\
& x_{N}-x_{N}^{s}=0 .
\end{aligned}
$$

(Quadratic) stage cost

Initial condition

System dynamics

Terminal constraint

At each sampling time $i$ :

- get the initial state $\bar{x}_{i}$

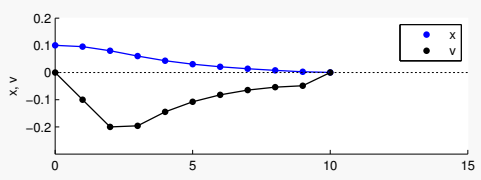

- solve the MPC OCP

- apply the first control $u_{0}^{\star}$

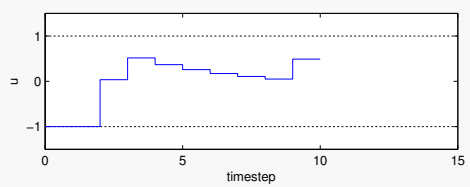




\section{Economic MPC and Stability Analysis}

MPC

Optimal Control Problem

$$
\begin{aligned}
\min _{x_{0}, u_{0}, \ldots, x_{N}} & \sum_{k=0}^{N-1} I\left(x_{k}, u_{k}\right) \\
\text { s.t. } & x_{0}-\bar{x}_{i}=0, \\
& x_{k+1}-f\left(x_{k}, u_{k}\right)=0, \\
& x_{N}-x_{N}^{s}=0 .
\end{aligned}
$$

(Quadratic) stage cost

Initial condition

System dynamics

Terminal constraint

At each sampling time $i$ :

- get the initial state $\bar{x}_{i}$

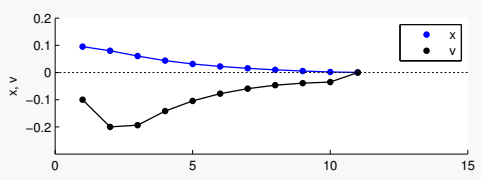

- solve the MPC OCP

- apply the first control $u_{0}^{\star}$

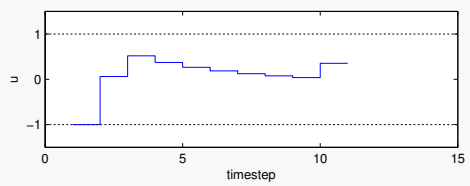




\section{Economic MPC and Stability Analysis}

MPC

Optimal Control Problem

$$
\begin{aligned}
\min _{x_{0}, u_{0}, \ldots, x_{N}} & \sum_{k=0}^{N-1} I\left(x_{k}, u_{k}\right) \\
\text { s.t. } & x_{0}-\bar{x}_{i}=0, \\
& x_{k+1}-f\left(x_{k}, u_{k}\right)=0, \\
& x_{N}-x_{N}^{s}=0 .
\end{aligned}
$$

(Quadratic) stage cost

Initial condition

System dynamics

Terminal constraint

At each sampling time $i$ :

- get the initial state $\bar{x}_{i}$

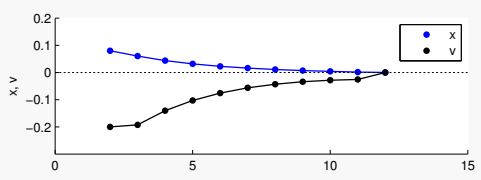

- solve the MPC OCP

- apply the first control $u_{0}^{\star}$

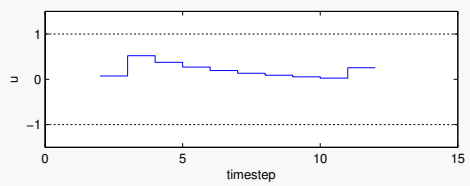




\section{Economic MPC and Stability Analysis}

MPC

Optimal Control Problem

$$
\begin{aligned}
\min _{x_{0}, u_{0}, \ldots, x_{N}} & \sum_{k=0}^{N-1} I\left(x_{k}, u_{k}\right) \\
\text { s.t. } & x_{0}-\bar{x}_{i}=0, \\
& x_{k+1}-f\left(x_{k}, u_{k}\right)=0, \\
& x_{N}-x_{N}^{s}=0 .
\end{aligned}
$$

(Quadratic) stage cost

Initial condition

System dynamics

Terminal constraint

At each sampling time $i$ :

- get the initial state $\bar{x}_{i}$

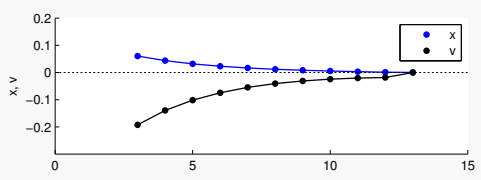

- solve the MPC OCP

- apply the first control $u_{0}^{\star}$

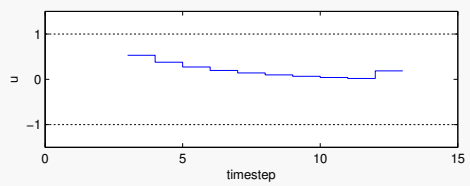




\section{Economic MPC and Stability Analysis}

MPC

Optimal Control Problem

$$
\begin{aligned}
\min _{x_{0}, u_{0}, \ldots, x_{N}} & \sum_{k=0}^{N-1} I\left(x_{k}, u_{k}\right) \\
\text { s.t. } & x_{0}-\bar{x}_{i}=0, \\
& x_{k+1}-f\left(x_{k}, u_{k}\right)=0, \\
& x_{N}-x_{N}^{s}=0 .
\end{aligned}
$$

(Quadratic) stage cost

Initial condition

System dynamics

Terminal constraint

At each sampling time $i$ :

- get the initial state $\bar{x}_{i}$

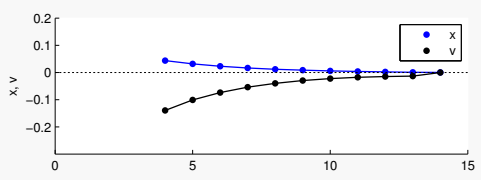

- solve the MPC OCP

- apply the first control $u_{0}^{\star}$

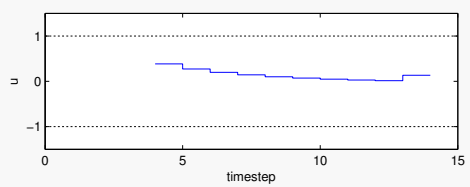




\section{Economic MPC and Stability Analysis}

\section{Lyapunov stability}

Tracking MPC: $I\left(x_{\mathrm{s}}, u_{\mathrm{s}}\right)=0$ and $\exists \alpha \in \mathcal{K}$ s.t. $\alpha\left(x-x_{\mathrm{s}}\right) \leq I(x, u), \forall u \in \mathbb{U}$ 


\section{Economic MPC and Stability Analysis}

\section{Lyapunov stability}

Tracking MPC: $I\left(x_{\mathrm{s}}, u_{\mathrm{s}}\right)=0$ and $\exists \alpha \in \mathcal{K}$ s.t. $\alpha\left(x-x_{\mathrm{s}}\right) \leq I(x, u), \forall u \in \mathbb{U}$

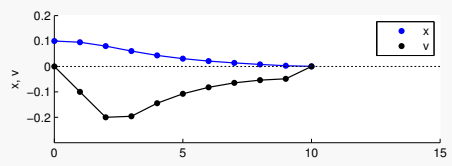

$$
V_{N}\left(\bar{x}_{i}\right)
$$

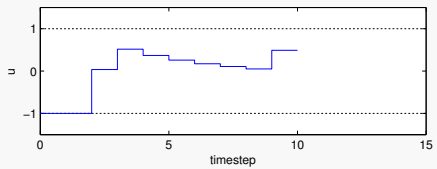




\section{Economic MPC and Stability Analysis}

\section{Lyapunov stability}

Tracking MPC: $I\left(x_{\mathrm{s}}, u_{\mathrm{s}}\right)=0$ and $\exists \alpha \in \mathcal{K}$ s.t. $\alpha\left(x-x_{\mathrm{s}}\right) \leq I(x, u), \forall u \in \mathbb{U}$

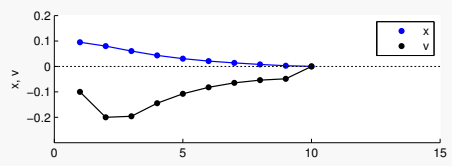

$$
V_{N-1}\left(f\left(\bar{x}_{i}, u_{0}^{\star}\right)\right)
$$

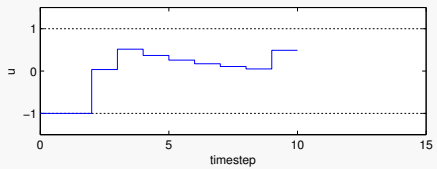




\section{Economic MPC and Stability Analysis}

\section{Lyapunov stability}

Tracking MPC: $I\left(x_{\mathrm{s}}, u_{\mathrm{s}}\right)=0$ and $\exists \alpha \in \mathcal{K}$ s.t. $\alpha\left(x-x_{\mathrm{s}}\right) \leq I(x, u), \forall u \in \mathbb{U}$

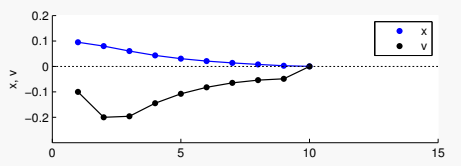

$$
V_{N-1}\left(f\left(\bar{x}_{i}, u_{0}^{\star}\right)\right)
$$

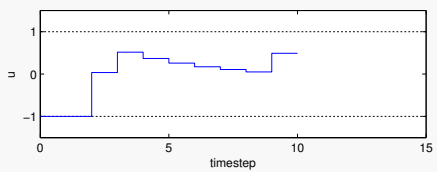

$$
V_{N-1}\left(f\left(\bar{x}_{i}, u_{0}^{\star}\right)\right)=V_{N}\left(\bar{x}_{i}\right)-I\left(\bar{x}_{i}, u_{0}^{\star}\right)
$$




\section{Economic MPC and Stability Analysis}

\section{Lyapunov stability}

Tracking MPC: $I\left(x_{\mathrm{s}}, u_{\mathrm{s}}\right)=0$ and $\exists \alpha \in \mathcal{K}$ s.t. $\alpha\left(x-x_{\mathrm{s}}\right) \leq I(x, u), \forall u \in \mathbb{U}$

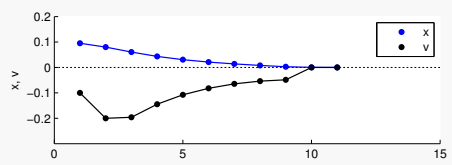

$$
V_{N-1}\left(f\left(\bar{x}_{i}, u_{0}^{\star}\right)\right)+I\left(x_{\mathrm{s}}, u_{\mathrm{s}}\right)
$$

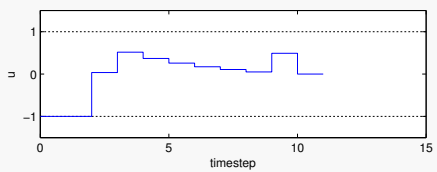

$$
\begin{aligned}
V_{N-1}\left(f\left(\bar{x}_{i}, u_{0}^{\star}\right)\right)= & V_{N}\left(\bar{x}_{i}\right)-I\left(\bar{x}_{i}, u_{0}^{\star}\right) \\
& V_{N}\left(\bar{x}_{i}\right)-I\left(\bar{x}_{i}, u_{0}^{\star}\right)+\underbrace{I\left(x_{\mathrm{s}}, u_{\mathrm{s}}\right)}_{=0}
\end{aligned}
$$




\section{Economic MPC and Stability Analysis}

\section{Lyapunov stability}

Tracking MPC: $I\left(x_{\mathrm{s}}, u_{\mathrm{s}}\right)=0$ and $\exists \alpha \in \mathcal{K}$ s.t. $\alpha\left(x-x_{\mathrm{s}}\right) \leq I(x, u), \forall u \in \mathbb{U}$

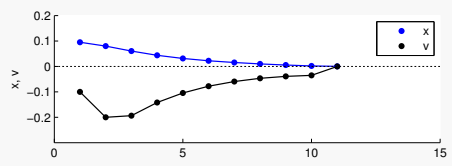

$$
V_{N}\left(f\left(\bar{x}_{i}, u_{0}^{\star}\right)\right)
$$

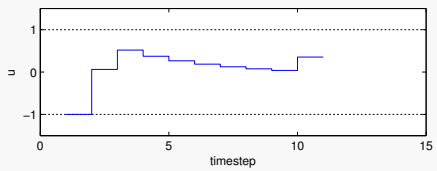

$$
\begin{aligned}
V_{N-1}\left(f\left(\bar{x}_{i}, u_{0}^{\star}\right)\right) & =V_{N}\left(\bar{x}_{i}\right)-I\left(\bar{x}_{i}, u_{0}^{\star}\right) \\
V_{N}\left(f\left(\bar{x}_{i}, u_{0}^{\star}\right)\right) & \leq V_{N}\left(\bar{x}_{i}\right)-I\left(\bar{x}_{i}, u_{0}^{\star}\right)+\underbrace{I\left(x_{\mathrm{s}}, u_{\mathrm{s}}\right)}_{=0}
\end{aligned}
$$




\section{Economic MPC and Stability Analysis}

\section{Lyapunov stability}

Tracking MPC: $I\left(x_{\mathrm{s}}, u_{\mathrm{s}}\right)=0$ and $\exists \alpha \in \mathcal{K}$ s.t. $\alpha\left(x-x_{\mathrm{s}}\right) \leq I(x, u), \forall u \in \mathbb{U}$

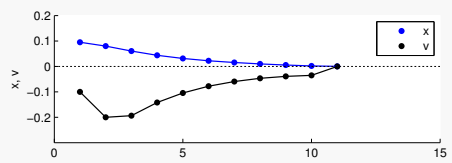

$$
V_{N}\left(f\left(\bar{x}_{i}, u_{0}^{\star}\right)\right)
$$

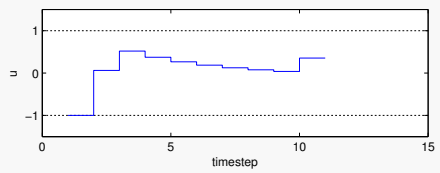

$$
\begin{aligned}
V_{N-1}\left(f\left(\bar{x}_{i}, u_{0}^{\star}\right)\right) & =V_{N}\left(\bar{x}_{i}\right)-I\left(\bar{x}_{i}, u_{0}^{\star}\right) \\
V_{N}\left(f\left(\bar{x}_{i}, u_{0}^{\star}\right)\right) & \leq V_{N}\left(\bar{x}_{i}\right)-I\left(\bar{x}_{i}, u_{0}^{\star}\right)+\underbrace{I\left(x_{\mathrm{s}}, u_{\mathrm{s}}\right)}_{=0}
\end{aligned}
$$

$$
V_{N}\left(f\left(\bar{x}_{i}, u_{0}^{\star}\right)\right)-V_{N}\left(\bar{x}_{i}\right) \leq-I\left(\bar{x}_{i}, u_{0}^{\star}\right)
$$


Do we always want to track? 
Economic MPC and Stability Analysis

Do we always want to track?

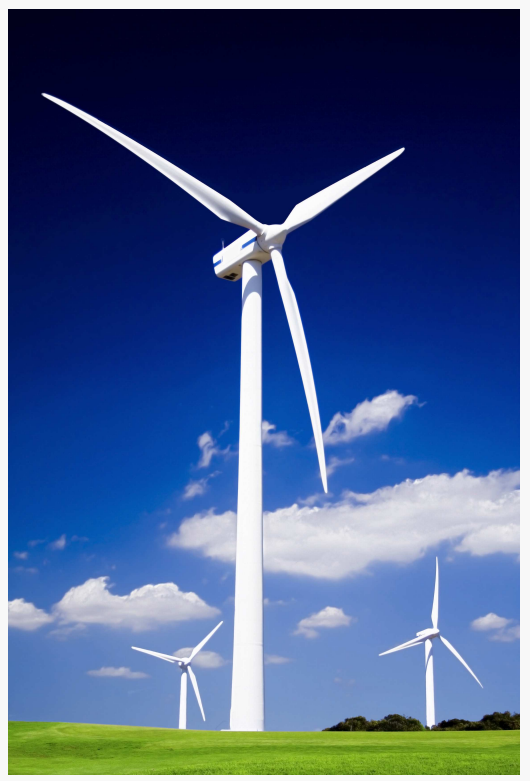


Do we always want to track?
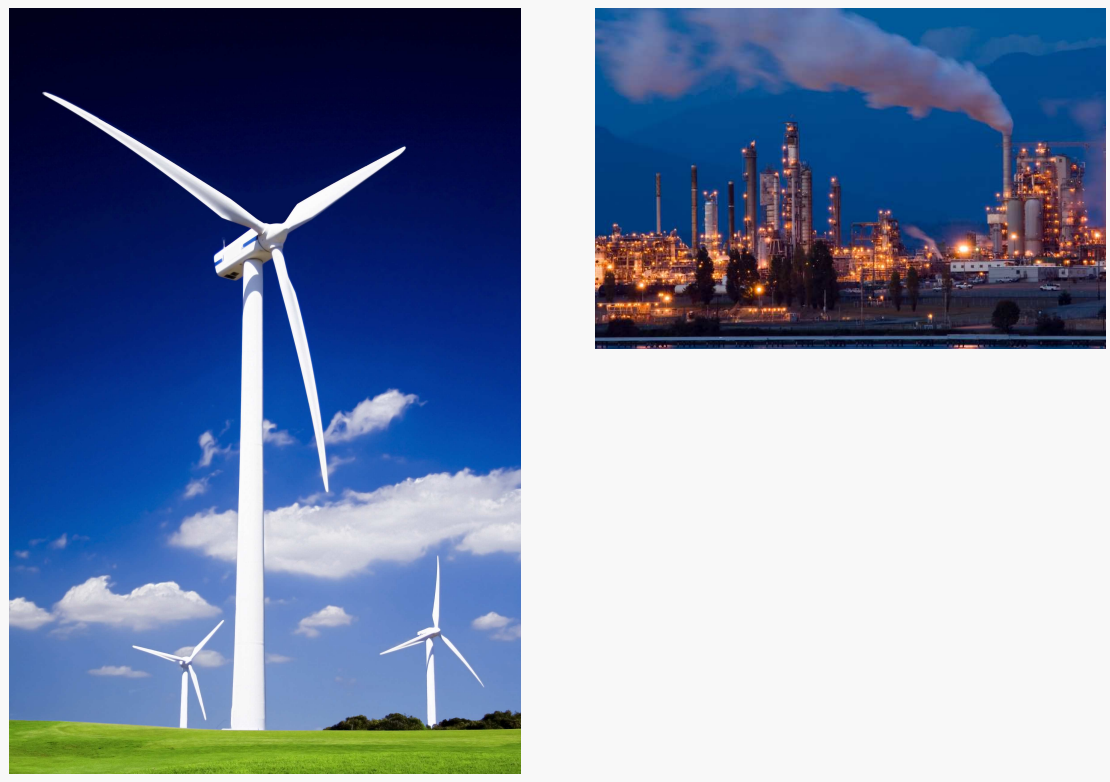
Do we always want to track?
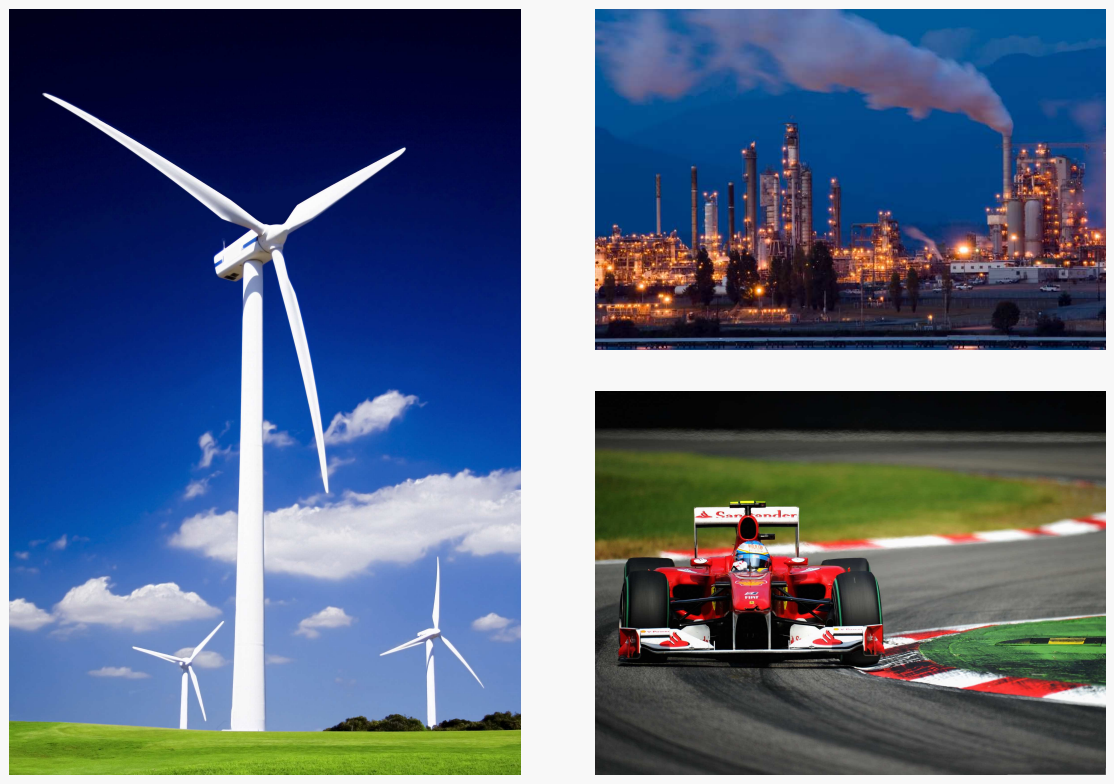
Do we always want to track?

No!
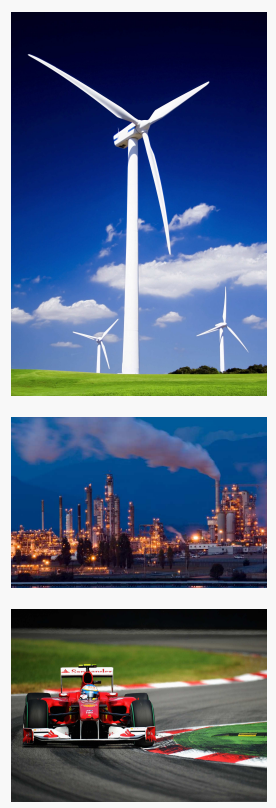
Do we always want to track?

No!

Then why do we track?
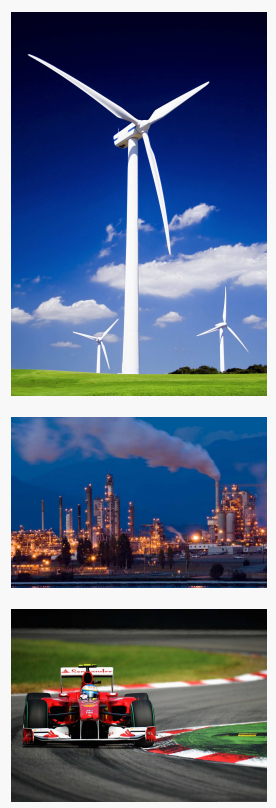
Do we always want to track?

No!

Then why do we track?

- It works
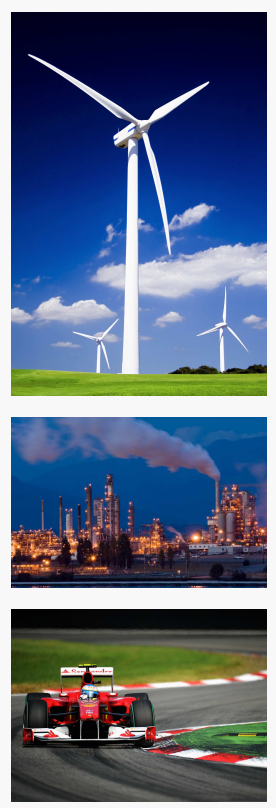
Do we always want to track?

No!

Then why do we track?

- It works

- We have been doing it since the 80 s
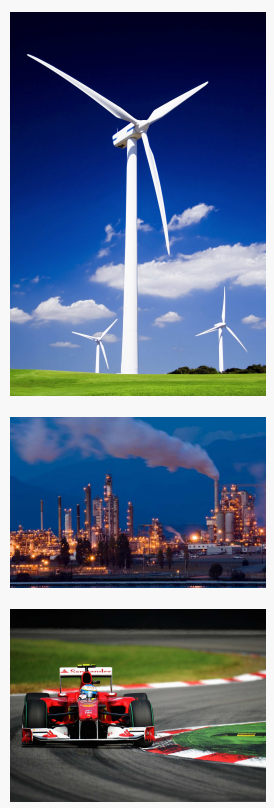
Do we always want to track?

No!

Then why do we track?

- It works

- We have been doing it since the $80 \mathrm{~s}$

- We have stability guarantees
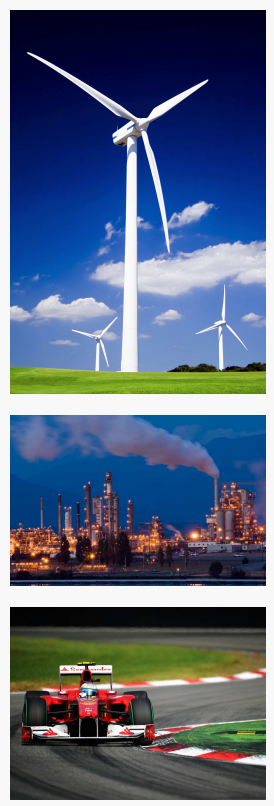
Do we always want to track?

No!

Then why do we track?

- It works

- We have been doing it since the $80 \mathrm{~s}$

- We have stability guarantees

What about Economic MPC?
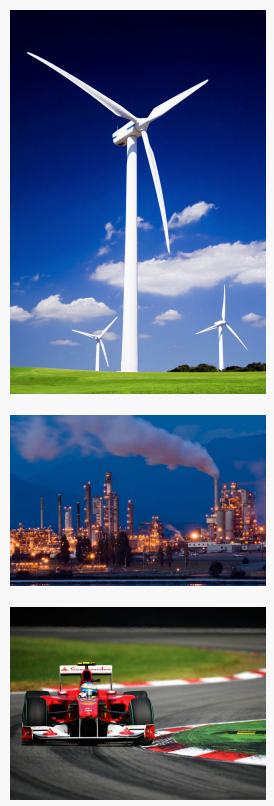
Do we always want to track?

No!

Then why do we track?

- It works

- We have been doing it since the $80 \mathrm{~s}$

- We have stability guarantees

What about Economic MPC?

- Difficult to prove stability (2008-)
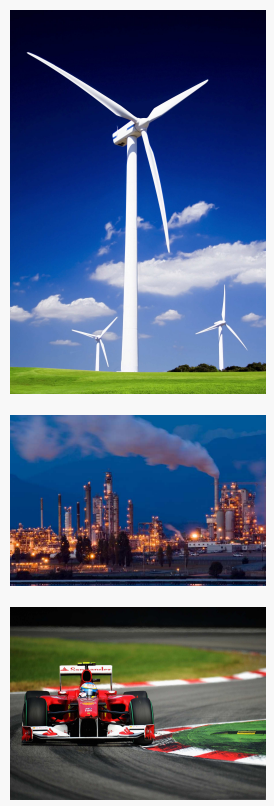
Do we always want to track?

No!

Then why do we track?

- It works

- We have been doing it since the $80 \mathrm{~s}$

- We have stability guarantees

What about Economic MPC?

- Difficult to prove stability (2008-)

- Increased "economic" gain
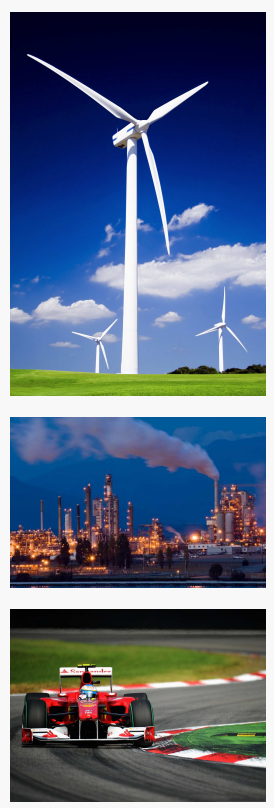


\section{Economic vs Tracking}




\section{Economic vs Tracking}

- Steady state: $x_{\mathrm{s}}=0=f(0,0)$ 


\section{Economic vs Tracking}

- Steady state: $x_{\mathrm{s}}=0=f(0,0)$

- Stage cost: Tracking vs Economic 


\section{Economic vs Tracking}

- Steady state: $x_{\mathrm{s}}=0=f(0,0)$

- Stage cost: Tracking vs Economic

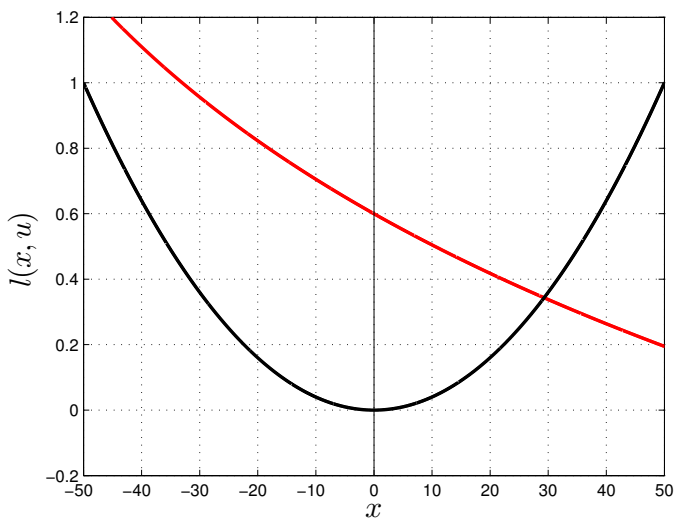




\section{Economic vs Tracking}

- Steady state: $x_{\mathrm{s}}=0=f(0,0)$

- Stage cost: Tracking vs Economic

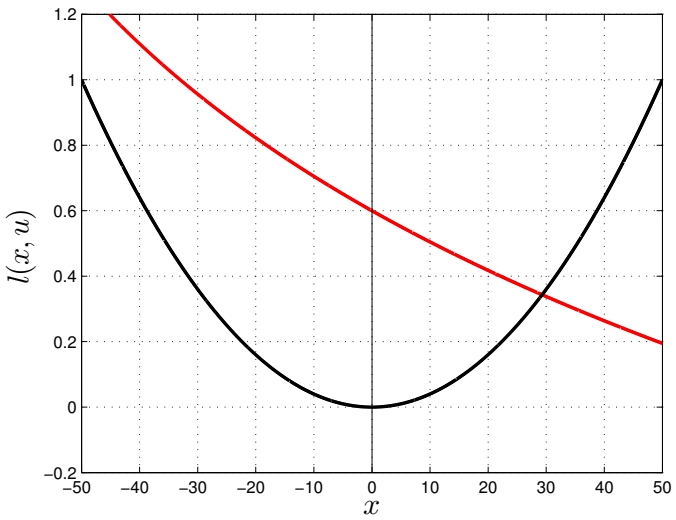

The classical stability theory does not apply! 


\section{Economic MPC and Stability Analysis}

\section{Economic Stage Cost}

Steady state: $\quad\left(x_{\mathrm{s}}, u_{\mathrm{s}}\right)=\min _{x, u} I(x, u)$ s.t. $x=f(x, u)$ 


\section{Economic MPC and Stability Analysis}

\section{Economic Stage Cost}

$$
\begin{gathered}
\text { Steady state: }\left(x_{\mathrm{s}}, u_{\mathrm{s}}\right)=\min _{x, u} I(x, u) \text { s.t. } x=f(x, u) \\
I(x, u) \quad I\left(x_{\mathrm{s}}, u_{\mathrm{s}}\right)
\end{gathered}
$$

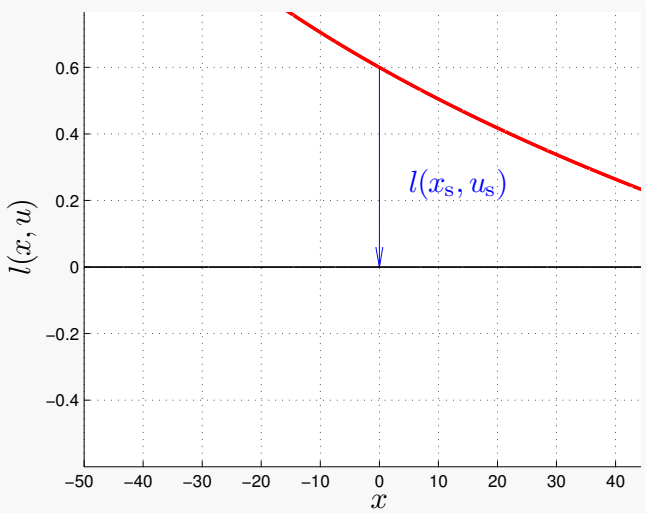




\section{Economic Stage Cost}

$$
\begin{gathered}
\text { Steady state: }\left(x_{\mathrm{s}}, u_{\mathrm{s}}\right)=\min _{x, u} I(x, u) \text { s.t. } x=f(x, u) \\
I(x, u)-I\left(x_{\mathrm{s}}, u_{\mathrm{s}}\right)
\end{gathered}
$$

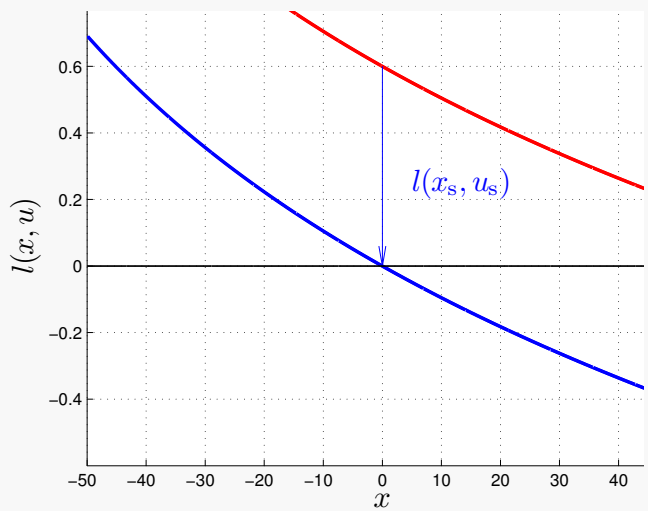




\section{Economic Stage Cost}

$$
\begin{gathered}
\text { Steady state: }\left(x_{\mathrm{s}}, u_{\mathrm{s}}\right)=\min _{x, u} I(x, u) \text { s.t. } x=f(x, u) \\
I(x, u)-I\left(x_{\mathrm{s}}, u_{\mathrm{s}}\right)
\end{gathered}
$$

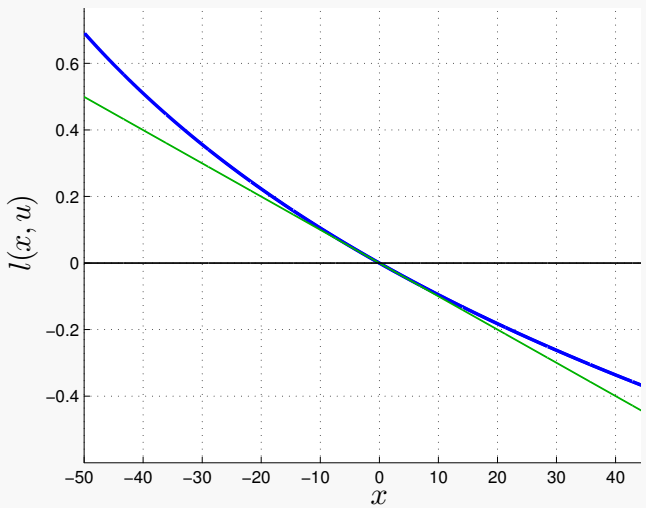




\section{Economic Stage Cost}

$$
\begin{aligned}
& \text { Steady state: }\left(x_{\mathrm{s}}, u_{\mathrm{s}}\right)=\min _{x, u} I(x, u) \text { s.t. } x=f(x, u) \\
& \qquad I(x, u)-I\left(x_{\mathrm{s}}, u_{\mathrm{s}}\right) \underbrace{\lambda_{\mathrm{s}}^{T}}_{\text {Lagrange multiplier }}(x-f(x, u))
\end{aligned}
$$

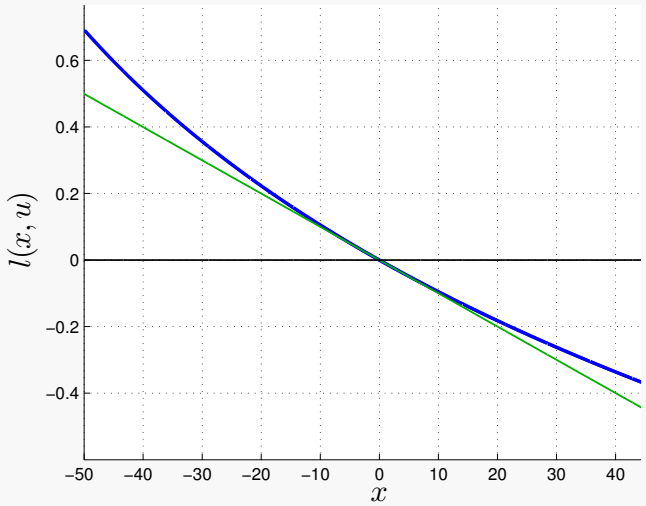




\section{Economic Stage Cost}

Steady state: $\quad\left(x_{\mathrm{s}}, u_{\mathrm{s}}\right)=\min _{x, u} I(x, u)$ s.t. $x=f(x, u)$

$$
I(x, u)-I\left(x_{\mathrm{s}}, u_{\mathrm{s}}\right)+\underbrace{\lambda_{\mathrm{s}}^{T}}_{\text {Lagrange multiplier }}(x-f(x, u))
$$

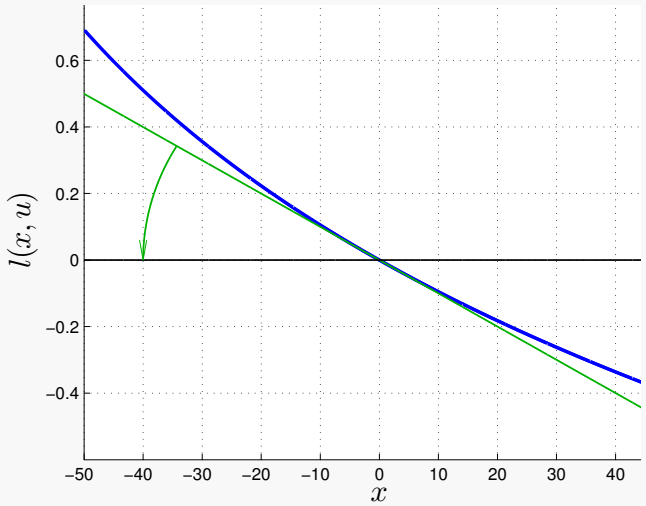




\section{Economic MPC and Stability Analysis}

\section{Economic Stage Cost}

Steady state: $\quad\left(x_{\mathrm{s}}, u_{\mathrm{s}}\right)=\min _{x, u} I(x, u)$ s.t. $x=f(x, u)$

$$
L(x, u)=I(x, u)-I\left(x_{\mathrm{s}}, u_{\mathrm{s}}\right)+\underbrace{\lambda_{\mathrm{s}}^{T}}_{\text {Lagrange multiplier }}(x-f(x, u))
$$

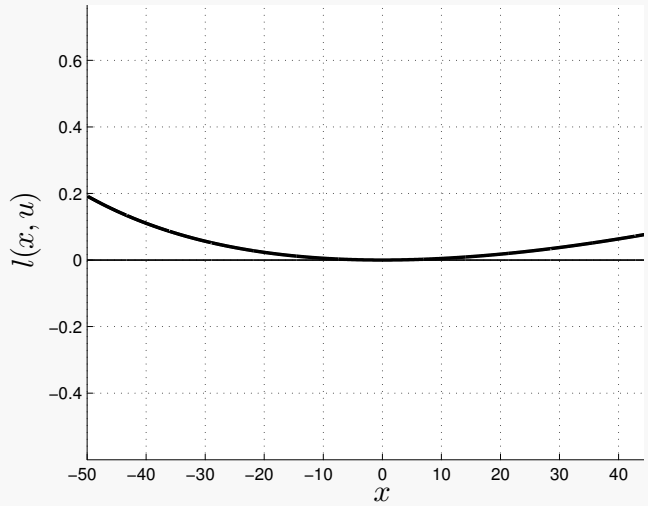




\section{Economic MPC and Stability Analysis}

\section{Economic Stage Cost}

$$
\text { Steady state: } \quad\left(x_{\mathrm{s}}, u_{\mathrm{s}}\right)=\min _{x, u} I(x, u) \text { s.t. } x=f(x, u)
$$
$\underbrace{\text { Rotated cost: }}_{\text {[Diehl et al. 2011] }} L(x, u)=I(x, u)-I\left(x_{\mathrm{s}}, u_{\mathrm{s}}\right)+\underbrace{\lambda_{\mathrm{s}}^{T}}_{\text {Lagrange multiplier }}(x-f(x, u))$

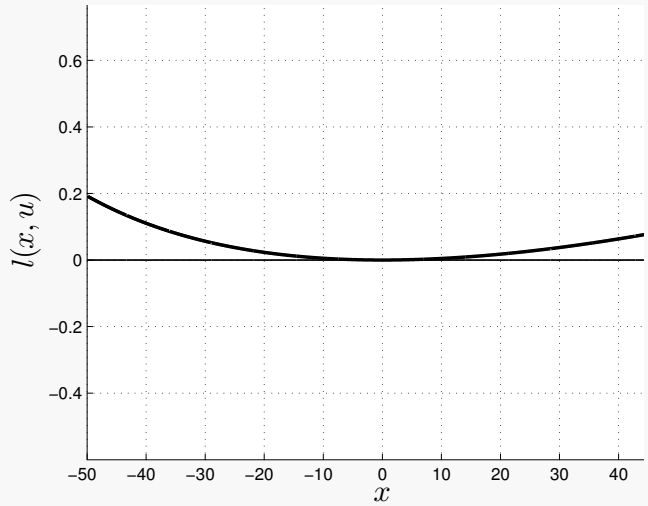




\section{Rotated MPC Problem}

$$
\begin{aligned}
\min _{x_{0}, u_{0}, \ldots, x_{N}} & \sum_{k=0}^{N-1} L\left(x_{k}, u_{k}\right) \\
\text { s.t. } & x_{0}-\bar{x}_{i}=0 \\
& x_{k+1}-f\left(x_{k}, u_{k}\right)=0, \\
& x_{N}-x_{N}^{s}=0 .
\end{aligned}
$$




\section{Rotated MPC Problem}

$$
\begin{array}{cl}
\min _{x_{0}, u_{0}, \ldots, x_{N}} & \sum_{k=0}^{N-1} L\left(x_{k}, u_{k}\right) \\
\text { s.t. } & x_{0}-\bar{x}_{i}=0 \\
& x_{k+1}-f\left(x_{k}, u_{k}\right)=0 \\
& x_{N}-x_{N}^{s}=0 .
\end{array}
$$

Rotated Problem $\equiv$ Original Problem

$$
\sum_{k=0}^{N-1} L(x, u)=\sum_{k=0}^{N-1} I(x, u)+\underbrace{\lambda_{\mathrm{s}}^{T} x_{0}-\lambda_{\mathrm{s}}^{T} x_{N}-(N-1) I\left(x_{\mathrm{s}}, u_{\mathrm{s}}\right)}_{\text {constant }}
$$




\section{Rotated MPC Problem}

$$
\begin{aligned}
\min _{x_{0}, u_{0}, \ldots, x_{N}} & \sum_{k=0}^{N-1} L\left(x_{k}, u_{k}\right) \\
\text { s.t. } & x_{0}-\bar{x}_{i}=0 \\
& x_{k+1}-f\left(x_{k}, u_{k}\right)=0 \\
& x_{N}-x_{N}^{s}=0 .
\end{aligned}
$$

Rotated Problem $\equiv$ Original Problem

$$
\sum_{k=0}^{N-1} L(x, u)=\sum_{k=0}^{N-1} I(x, u)+\underbrace{\lambda_{\mathrm{s}}^{T} x_{0}-\lambda_{\mathrm{s}}^{T} x_{N}-(N-1) I\left(x_{\mathrm{s}}, u_{\mathrm{s}}\right)}_{\text {constant }}
$$

If

$$
\alpha(x) \leq L(x, u), \quad \alpha \in \mathcal{K}
$$

the previous stability proof holds! [Diehl et al. 2011] 


\section{Economic MPC and Stability Analysis}

Generalization [Amrit et al. 2011]

Nonlinear rotating function: $\lambda(x)$ 


\section{Economic MPC and Stability Analysis}

Generalization [Amrit et al. 2011]

Nonlinear rotating function: $\lambda(x)$

New rotated cost: $L(x, u)=I(x, u)-I\left(x_{\mathrm{s}}, u_{\mathrm{s}}\right)+\lambda(x)-\lambda(f(x, u))$ 
Generalization [Amrit et al. 2011]

Nonlinear rotating function: $\lambda(x)$

New rotated cost: $L(x, u)=I(x, u)-I\left(x_{\mathrm{s}}, u_{\mathrm{s}}\right)+\lambda(x)-\lambda(f(x, u))$

What conditions on $\lambda(x)$ ? 
Generalization [Amrit et al. 2011]

Nonlinear rotating function: $\lambda(x)$

New rotated cost: $L(x, u)=I(x, u)-I\left(x_{\mathrm{s}}, u_{\mathrm{s}}\right)+\lambda(x)-\lambda(f(x, u))$

\section{What conditions on $\lambda(x)$ ?}

\section{Strict Dissipativity}

System strictly dissipative wrt the supply rate $s(x, u)$ if $\exists \lambda(x): \mathbb{X} \rightarrow \mathbb{R}$

$$
\lambda(f(x, u))-\lambda(x) \leq-\rho\left(x-x_{\mathrm{s}}\right)+s(x, u),
$$

$$
\forall(x, u) \in \mathbb{X} \times \mathbb{U}
$$

We are interested in $s(x, u)=I(x, u)-I\left(x_{\mathrm{s}}, u_{\mathrm{s}}\right)$ 
Generalization [Amrit et al. 2011]

Nonlinear rotating function: $\lambda(x)$

New rotated cost: $L(x, u)=I(x, u)-I\left(x_{\mathrm{s}}, u_{\mathrm{s}}\right)+\lambda(x)-\lambda(f(x, u))$

\section{What conditions on $\lambda(x)$ ?}

\section{Strict Dissipativity}

System strictly dissipative wrt the supply rate $s(x, u)$ if $\exists \lambda(x): \mathbb{X} \rightarrow \mathbb{R}$

$$
\lambda(f(x, u))-\lambda(x) \leq-\rho\left(x-x_{\mathrm{s}}\right)+s(x, u),
$$

$$
\forall(x, u) \in \mathbb{X} \times \mathbb{U}
$$

We are interested in $s(x, u)=I(x, u)-I\left(x_{\mathrm{s}}, u_{\mathrm{s}}\right)$

This entails

$$
L(x, u)=I(x, u)-I\left(x_{\mathrm{s}}, u_{\mathrm{s}}\right)+\lambda(x)-\lambda(f(x, u)) \geq \rho\left(x-x_{\mathrm{s}}\right)
$$


(1) Economic MPC and Stability Analysis

(2) The Linear Quadratic Case 


\section{LQ-EMPC}

Consider a linear MPC problem

$$
\begin{aligned}
\mathcal{P}_{N}\left(A, B, Q, R, S, P_{N}\right)=\underset{x_{0}, u_{0}, \ldots, x_{N}}{\operatorname{argmin}} & \sum_{k=0}^{N-1}\left[\begin{array}{l}
x_{k} \\
u_{k}
\end{array}\right]^{T} H\left[\begin{array}{l}
x_{k} \\
u_{k}
\end{array}\right]+x_{N}^{T} P_{N} x_{N} \\
\text { s.t. } \quad & x_{0}-\bar{x}_{i}=0, \\
& x_{k+1}-A x_{k}-B u_{k}=0 .
\end{aligned}
$$

with

$$
H=\left[\begin{array}{ll}
Q & S^{T} \\
S & R
\end{array}\right]
$$




\section{LQ-EMPC}

Consider a linear MPC problem

$$
\begin{aligned}
\mathcal{P}_{N}\left(A, B, Q, R, S, P_{N}\right)=\underset{x_{0}, u_{0}, \ldots, x_{N}}{\operatorname{argmin}} & \sum_{k=0}^{N-1}\left[\begin{array}{c}
x_{k} \\
u_{k}
\end{array}\right]^{T} H\left[\begin{array}{l}
x_{k} \\
u_{k}
\end{array}\right]+x_{N}^{T} P_{N} x_{N} \\
\text { s.t. } \quad & x_{0}-\bar{x}_{i}=0, \\
& x_{k+1}-A x_{k}-B u_{k}=0 .
\end{aligned}
$$

with

$$
H=\left[\begin{array}{ll}
Q & S^{T} \\
S & R
\end{array}\right]
$$

- If $H \nsucc 0$ this is an Economic MPC problem 


\section{LQ-EMPC}

Consider a linear MPC problem

$$
\begin{aligned}
\mathcal{P}_{N}\left(A, B, Q, R, S, P_{N}\right)=\underset{x_{0}, u_{0}, \ldots, x_{N}}{\operatorname{argmin}} & \sum_{k=0}^{N-1}\left[\begin{array}{c}
x_{k} \\
u_{k}
\end{array}\right]^{T} H\left[\begin{array}{l}
x_{k} \\
u_{k}
\end{array}\right]+x_{N}^{T} P_{N} x_{N} \\
\text { s.t. } \quad & x_{0}-\bar{x}_{i}=0, \\
& x_{k+1}-A x_{k}-B u_{k}=0 .
\end{aligned}
$$

with

$$
H=\left[\begin{array}{ll}
Q & S^{T} \\
S & R
\end{array}\right]
$$

- If $H \nsucc 0$ this is an Economic MPC problem

- When is it stabilizing? 


\section{Inifinite Horizon Case}




\section{Inifinite Horizon Case}

This is an (indefinite) LQR

$$
\begin{aligned}
\mathcal{D}(A, B, Q, R, S):=\{(P, K) \mid & Q+A^{T} P A-P-\left(S^{T}+A^{T} P B\right) K=0, \\
& K=\left(R+B^{T} P B\right)^{-1}\left(S+B^{T} P A\right), \\
& \rho(A-B K)<1\}
\end{aligned}
$$




\section{Inifinite Horizon Case}

This is an (indefinite) LQR

$$
\begin{aligned}
\mathcal{D}(A, B, Q, R, S):=\{(P, K) \mid & Q+A^{T} P A-P-\left(S^{T}+A^{T} P B\right) K=0, \\
& K=\left(R+B^{T} P B\right)^{-1}\left(S+B^{T} P A\right), \\
& \rho(A-B K)<1\}
\end{aligned}
$$

Stability if (strict dissipativity for the LQ case):

$$
\exists \bar{P} \text { s.t. } \quad M=\left[\begin{array}{ll}
Q+A^{T} \bar{P} A-\bar{P} & S^{T}+A^{T} \bar{P} B \\
S+B^{T} \bar{P} A & R+B^{T} \bar{P} B
\end{array}\right] \succ 0
$$




\section{Inifinite Horizon Case}

This is an (indefinite) LQR

$$
\begin{aligned}
\mathcal{D}(A, B, Q, R, S):=\{(P, K) \mid & Q+A^{T} P A-P-\left(S^{T}+A^{T} P B\right) K=0, \\
& K=\left(R+B^{T} P B\right)^{-1}\left(S+B^{T} P A\right), \\
& \rho(A-B K)<1\}
\end{aligned}
$$

Stability if (strict dissipativity for the LQ case):

$$
\exists \bar{P} \text { s.t. } \quad M=\left[\begin{array}{ll}
Q+A^{T} \bar{P} A-\bar{P} & S^{T}+A^{T} \bar{P} B \\
S+B^{T} \bar{P} A & R+B^{T} \bar{P} B
\end{array}\right] \succ 0
$$

One can enforce stability by adding $\left[\begin{array}{l}x \\ u\end{array}\right]^{T} T\left[\begin{array}{l}x \\ u\end{array}\right]$ to the cost and solving

$$
\min _{P, T}\|T\|^{2} \quad \text { s.t. } M+T \succeq 0,
$$


One can modify the cost-to-go without changing the problem

$$
\mathcal{P}_{\infty}(A, B, Q, R, S)=\mathcal{P}_{\infty}\left(A, B, Q_{\bar{P}}, R_{\bar{P}}, S_{\bar{P}}\right),
$$

with

$$
\begin{aligned}
Q_{\bar{P}} & =Q+A^{T} \bar{P} A-\bar{P} \\
R_{\bar{P}} & =R+B^{T} \bar{P} B \\
S_{\bar{P}} & =S+B^{T} \bar{P} A \\
P_{\bar{P}} & =P-\bar{P}
\end{aligned}
$$


One can modify the cost-to-go without changing the problem

$$
\mathcal{P}_{\infty}(A, B, Q, R, S)=\mathcal{P}_{\infty}\left(A, B, Q_{\bar{P}}, R_{\bar{P}}, S_{\bar{P}}\right)
$$

with

$$
\begin{aligned}
Q_{\bar{P}} & =Q+A^{T} \bar{P} A-\bar{P} \\
R_{\bar{P}} & =R+B^{T} \bar{P} B \\
S_{\bar{P}} & =S+B^{T} \bar{P} A \\
P_{\bar{P}} & =P-\bar{P}
\end{aligned}
$$

NOTE:

- $K_{\bar{P}}=K$, i.e. the feedback matrix is unchanged! 
One can modify the cost-to-go without changing the problem

$$
\mathcal{P}_{\infty}(A, B, Q, R, S)=\mathcal{P}_{\infty}\left(A, B, Q_{\bar{P}}, R_{\bar{P}}, S_{\bar{P}}\right)
$$

with

$$
\begin{aligned}
Q_{\bar{P}} & =Q+A^{T} \bar{P} A-\bar{P} \\
R_{\bar{P}} & =R+B^{T} \bar{P} B \\
S_{\bar{P}} & =S+B^{T} \bar{P} A \\
P_{\bar{P}} & =P-\bar{P}
\end{aligned}
$$

NOTE:

- $K_{\bar{P}}=K$, i.e. the feedback matrix is unchanged!

- $P_{P}=0$, i.e. the cost-to-go can be zero! 


\section{One Can Pre-stabilize the System}

i.e. use $u_{k}=v_{k}-\bar{K} x_{k}$ 


\section{One Can Pre-stabilize the System}

i.e. use $u_{k}=v_{k}-\bar{K} x_{k}$

Then

$$
\mathcal{P}_{\infty}(A, B, Q, R, S)=\mathcal{P}_{\infty}\left(A_{\bar{K}}, B, Q_{\bar{K}}, R, S_{\bar{K}}\right)
$$

with

$$
\begin{aligned}
A_{\bar{K}} & =A-B \bar{K} \\
Q_{\bar{K}} & =Q-S^{T} \bar{K}-\bar{K}^{T} S+\bar{K}^{T} R \bar{K} \\
S_{\bar{K}} & =S-R \bar{K} \\
P_{\bar{K}} & =P
\end{aligned}
$$




\section{One Can Pre-stabilize the System}

i.e. use $u_{k}=v_{k}-\bar{K} x_{k}$

Then

$$
\mathcal{P}_{\infty}(A, B, Q, R, S)=\mathcal{P}_{\infty}\left(A_{\bar{K}}, B, Q_{\bar{K}}, R, S_{\bar{K}}\right),
$$

with

$$
\begin{aligned}
A_{\bar{K}} & =A-B \bar{K} \\
Q_{\bar{K}} & =Q-S^{T} \bar{K}-\bar{K}^{T} S+\bar{K}^{T} R \bar{K} \\
S_{\bar{K}} & =S-R \bar{K} \\
P_{\bar{K}} & =P
\end{aligned}
$$

If one uses the optimal feedback gain $\bar{K}=K$ : 


\section{One Can Pre-stabilize the System}

i.e. use $u_{k}=v_{k}-\bar{K} x_{k}$

Then

$$
\mathcal{P}_{\infty}(A, B, Q, R, S)=\mathcal{P}_{\infty}\left(A_{\bar{K}}, B, Q_{\bar{K}}, R, S_{\bar{K}}\right),
$$

with

$$
\begin{aligned}
A_{\bar{K}} & =A-B \bar{K} \\
Q_{\bar{K}} & =Q-S^{T} \bar{K}-\bar{K}^{T} S+\bar{K}^{T} R \bar{K} \\
S_{\bar{K}} & =S-R \bar{K} \\
P_{\bar{K}} & =P
\end{aligned}
$$

If one uses the optimal feedback gain $\bar{K}=K$ :

- $K_{K}=0$ 


\section{One Can Pre-stabilize the System}

i.e. use $u_{k}=v_{k}-\bar{K} x_{k}$

Then

$$
\mathcal{P}_{\infty}(A, B, Q, R, S)=\mathcal{P}_{\infty}\left(A_{\bar{K}}, B, Q_{\bar{K}}, R, S_{\bar{K}}\right)
$$

with

$$
\begin{aligned}
A_{\bar{K}} & =A-B \bar{K} \\
Q_{\bar{K}} & =Q-S^{T} \bar{K}-\bar{K}^{T} S+\bar{K}^{T} R \bar{K} \\
S_{\bar{K}} & =S-R \bar{K} \\
P_{\bar{K}} & =P
\end{aligned}
$$

If one uses the optimal feedback gain $\bar{K}=K$ :

- $K_{K}=0$

- The DARE becomes a Lyapunov Equation: $Q_{K}+A_{K}^{T} P A_{K}-P=0$ 


\section{One Can Pre-stabilize the System}

i.e. use $u_{k}=v_{k}-\bar{K} x_{k}$

Then

$$
\mathcal{P}_{\infty}(A, B, Q, R, S)=\mathcal{P}_{\infty}\left(A_{\bar{K}}, B, Q_{\bar{K}}, R, S_{\bar{K}}\right)
$$

with

$$
\begin{aligned}
A_{\bar{K}} & =A-B \bar{K} \\
Q_{\bar{K}} & =Q-S^{T} \bar{K}-\bar{K}^{T} S+\bar{K}^{T} R \bar{K} \\
S_{\bar{K}} & =S-R \bar{K} \\
P_{\bar{K}} & =P
\end{aligned}
$$

If one uses the optimal feedback gain $\bar{K}=K$ :

- $K_{K}=0$

- The DARE becomes a Lyapunov Equation: $Q_{K}+A_{K}^{T} P A_{K}-P=0$

- $R$ can be replaced by any $R_{K}(\succ 0)$ 


\section{Positive Definite LQR Formulation}

Lyapunov Stability Theorem

If $\rho\left(A_{K}\right)<1$, then

$$
\forall Q_{\mathrm{L}} \succ 0
$$$$
\exists P_{\mathrm{L}} \succ 0 \text { s.t. }
$$$$
Q_{\mathrm{L}}+A_{K}^{T} P_{\mathrm{L}} A_{K}-P_{\mathrm{L}}=0
$$ 


\section{Positive Definite LQR Formulation}

Lyapunov Stability Theorem

If $\rho\left(A_{K}\right)<1$, then

$$
\forall Q_{\mathrm{L}} \succ 0 \quad \exists P_{\mathrm{L}} \succ 0 \text { s.t. } \quad Q_{\mathrm{L}}+A_{K}^{T} P_{\mathrm{L}} A_{K}-P_{\mathrm{L}}=0
$$

This is also the DARE for $\left(A_{K}, B\right) \Rightarrow$ Choose $Q_{K, P_{\mathrm{L}}}=Q_{\mathrm{L}} \succ 0$ 


\section{Positive Definite LQR Formulation}

\section{Lyapunov Stability Theorem}

If $\rho\left(A_{K}\right)<1$, then

$$
\forall Q_{\mathrm{L}} \succ 0 \quad \exists P_{\mathrm{L}} \succ 0 \text { s.t. } \quad Q_{\mathrm{L}}+A_{K}^{T} P_{\mathrm{L}} A_{K}-P_{\mathrm{L}}=0
$$

This is also the DARE for $\left(A_{K}, B\right) \Rightarrow$ Choose $Q_{K, P_{\mathrm{L}}}=Q_{\mathrm{L}} \succ 0$ Then

$$
\begin{aligned}
& P_{K, P_{\mathrm{L}}}=P-P_{\mathrm{L}} \\
& R_{K, P_{\mathrm{L}}}=R_{K}+B^{T} P_{\mathrm{L}} B \\
& S_{K, P_{\mathrm{L}}}=S_{K}+B^{T} P_{\mathrm{L}} A
\end{aligned}
$$




\section{Positive Definite LQR Formulation}

\section{Lyapunov Stability Theorem}

If $\rho\left(A_{K}\right)<1$, then

$$
\forall Q_{\mathrm{L}} \succ 0 \quad \exists P_{\mathrm{L}} \succ 0 \text { s.t. } \quad Q_{\mathrm{L}}+A_{K}^{T} P_{\mathrm{L}} A_{K}-P_{\mathrm{L}}=0
$$

This is also the DARE for $\left(A_{K}, B\right) \Rightarrow$ Choose $Q_{K, P_{\mathrm{L}}}=Q_{\mathrm{L}} \succ 0$ Then

$$
\begin{aligned}
& P_{K, P_{\mathrm{L}}}=P-P_{\mathrm{L}} \\
& R_{K, P_{\mathrm{L}}}=R_{K}+B^{T} P_{\mathrm{L}} B \\
& S_{K, P_{\mathrm{L}}}=S_{K}+B^{T} P_{\mathrm{L}} A
\end{aligned}
$$

$R_{K}$ can be chosen arbitrarily large $\Rightarrow$ Pos. def. LQR for $\left(A_{K}, B\right)$. 


\section{Positive Definite LQR Formulation}

\section{Lyapunov Stability Theorem}

If $\rho\left(A_{K}\right)<1$, then

$$
\forall Q_{\mathrm{L}} \succ 0 \quad \exists P_{\mathrm{L}} \succ 0 \text { s.t. } \quad Q_{\mathrm{L}}+A_{K}^{T} P_{\mathrm{L}} A_{K}-P_{\mathrm{L}}=0
$$

This is also the DARE for $\left(A_{K}, B\right) \Rightarrow$ Choose $Q_{K, P_{\mathrm{L}}}=Q_{\mathrm{L}} \succ 0$

Then

$$
\begin{aligned}
& P_{K, P_{\mathrm{L}}}=P-P_{\mathrm{L}} \\
& R_{K, P_{\mathrm{L}}}=R_{K}+B^{T} P_{\mathrm{L}} B \\
& S_{K, P_{\mathrm{L}}}=S_{K}+B^{T} P_{\mathrm{L}} A
\end{aligned}
$$

$R_{K}$ can be chosen arbitrarily large $\Rightarrow$ Pos. def. LQR for $\left(A_{K}, B\right)$.

Pos. def. is preserved when transforming back to $(A, B)$ 


\section{What About MPC?}

In the unconstrained case, the solution is given by the Discrete Riccati Equation (DRE)

$$
\begin{aligned}
\mathcal{R}_{N}\left(A, B, Q, R, S, P_{N}\right)=\{ & \left(P_{0}, P_{1}, \ldots, P_{N}, K_{0}, \ldots, K_{N-1}\right) \mid \\
& P_{k-1}=Q+A^{T} P_{k} A-\left(S^{T}+A^{T} P_{k} B\right) K_{k-1} \\
& \left.K_{k-1}=\left(R+B^{T} P_{k} B\right)^{-1}\left(S+B^{T} P_{k} A\right)\right\}
\end{aligned}
$$




\section{What About MPC?}

In the unconstrained case, the solution is given by the Discrete Riccati Equation (DRE)

$$
\begin{aligned}
\mathcal{R}_{N}\left(A, B, Q, R, S, P_{N}\right)=\{ & \left(P_{0}, P_{1}, \ldots, P_{N}, K_{0}, \ldots, K_{N-1}\right) \mid \\
& P_{k-1}=Q+A^{T} P_{k} A-\left(S^{T}+A^{T} P_{k} B\right) K_{k-1} \\
& \left.K_{k-1}=\left(R+B^{T} P_{k} B\right)^{-1}\left(S+B^{T} P_{k} A\right)\right\}
\end{aligned}
$$

DRE equivalence

$$
\mathcal{R}_{N}\left(A, B, Q, R, S, P_{N}\right)=\mathcal{R}_{N}\left(A, B, \tilde{Q}, \tilde{R}, \tilde{S}, \tilde{P}_{N}\right)
$$

if

$$
\begin{aligned}
& \tilde{P}_{N}-\tilde{P}=P_{N}-P, \\
& \tilde{R}+B^{T} \tilde{P} B=R+B^{T} P B, \\
& \tilde{S}+B^{T} \tilde{P} A=S+B^{T} P A
\end{aligned}
$$

with $P$ and $\tilde{P}$ computed from $\mathcal{D}(A, B, Q, R, S)$ and $\mathcal{D}(A, B, \tilde{Q}, \tilde{R}, \tilde{S})$. 


\section{A Practical View on the Problem}

Solve the following SDP

$$
\begin{array}{ll}
\min _{\tilde{P}, \tilde{Q}, \tilde{R}, \tilde{S}, \tilde{H}} & \|\tilde{P}-I\|^{2}+\|\tilde{H}-I\|^{2} \\
\text { s.t. } & \tilde{H}=\left[\begin{array}{cc}
\tilde{Q} & \tilde{S}^{T} \\
\tilde{S} & \tilde{R}
\end{array}\right] \\
& \tilde{H} \succeq 0 \\
& \tilde{P} \succeq 0 \\
& \tilde{Q}+A^{T} \tilde{P} A-\tilde{P}-\left(\tilde{S}^{T}+A^{T} \tilde{P} B\right) K=0, \\
& \left(\tilde{R}+B^{T} \tilde{P} B\right) K-\left(\tilde{S}+B^{T} \tilde{P} A\right)=0, \\
& \tilde{R}+B^{T} \tilde{P} B=R+B^{T} P B, \\
& \tilde{S}+B^{T} \tilde{P} A=S+B^{T} P A .
\end{array}
$$

This problem is convex! 
(1) Economic MPC and Stability Analysis

(2) The Linear Quadratic Case

(3) Approximated EMPC with stability guarantees 


\section{Look at the Steady State Properties}

Lagrangian of steady state problem

$$
\mathcal{L}=I(x, u)-\lambda^{T}(x-f(x, u))
$$

Lagrangian Hessian:

$$
H=\left[\begin{array}{ll}
Q & S^{T} \\
S & R
\end{array}\right]=\left.\frac{\partial^{2} \mathcal{L}}{\partial(x, u)^{2}}\right|_{x_{\mathrm{s}}, u_{\mathrm{S}}}
$$

Local linearization

$$
A=\left.\frac{\partial f(x, u)}{\partial x}\right|_{x_{\mathrm{s}}, u_{\mathrm{s}}} \quad B=\left.\frac{\partial f(x, u)}{\partial u}\right|_{\chi_{\mathrm{s}}, u_{\mathrm{s}}}
$$




\section{Look at the Steady State Properties}

Lagrangian of steady state problem

$$
\mathcal{L}=I(x, u)-\lambda^{T}(x-f(x, u))
$$

Lagrangian Hessian:

$$
H=\left[\begin{array}{ll}
Q & S^{T} \\
S & R
\end{array}\right]=\left.\frac{\partial^{2} \mathcal{L}}{\partial(x, u)^{2}}\right|_{x_{\mathrm{s}}, u_{\mathrm{S}}}
$$

Local linearization

$$
A=\left.\frac{\partial f(x, u)}{\partial x}\right|_{x_{\mathrm{s}}, u_{\mathrm{s}}} \quad B=\left.\frac{\partial f(x, u)}{\partial u}\right|_{x_{\mathrm{s}}, u_{\mathrm{s}}}
$$

Problem: $H \nsucc 0$ is indefinite! 


\section{Look at the Steady State Properties}

Lagrangian of steady state problem

$$
\mathcal{L}=I(x, u)-\lambda^{T}(x-f(x, u))
$$

Lagrangian Hessian:

$$
H=\left[\begin{array}{cc}
Q & S^{T} \\
S & R
\end{array}\right]=\left.\frac{\partial^{2} \mathcal{L}}{\partial(x, u)^{2}}\right|_{x_{\mathrm{s}}, u_{\mathrm{S}}}
$$

Local linearization

$$
A=\left.\frac{\partial f(x, u)}{\partial x}\right|_{x_{\mathrm{s}}, u_{\mathrm{s}}} \quad B=\left.\frac{\partial f(x, u)}{\partial u}\right|_{x_{\mathrm{s}}, u_{\mathrm{s}}}
$$

Problem: $H \nsucc 0$ is indefinite! Solution: 


\section{Look at the Steady State Properties}

Lagrangian of steady state problem

$$
\mathcal{L}=I(x, u)-\lambda^{T}(x-f(x, u))
$$

Lagrangian Hessian:

$$
H=\left[\begin{array}{ll}
Q & S^{T} \\
S & R
\end{array}\right]=\left.\frac{\partial^{2} \mathcal{L}}{\partial(x, u)^{2}}\right|_{x_{\mathrm{s}}, u_{\mathrm{S}}}
$$

Local linearization

$$
A=\left.\frac{\partial f(x, u)}{\partial x}\right|_{x_{\mathrm{s}}, u_{\mathrm{s}}} \quad B=\left.\frac{\partial f(x, u)}{\partial u}\right|_{x_{\mathrm{s}}, u_{\mathrm{s}}}
$$

Problem: $H \nsucc 0$ is indefinite! Solution:

- Check for stability of the local LQ approximation 


\section{Look at the Steady State Properties}

Lagrangian of steady state problem

$$
\mathcal{L}=I(x, u)-\lambda^{T}(x-f(x, u))
$$

Lagrangian Hessian:

$$
H=\left[\begin{array}{ll}
Q & S^{T} \\
S & R
\end{array}\right]=\left.\frac{\partial^{2} \mathcal{L}}{\partial(x, u)^{2}}\right|_{x_{\mathrm{s}}, u_{\mathrm{S}}}
$$

Local linearization

$$
A=\left.\frac{\partial f(x, u)}{\partial x}\right|_{x_{\mathrm{s}}, u_{\mathrm{s}}} \quad B=\left.\frac{\partial f(x, u)}{\partial u}\right|_{x_{\mathrm{s}}, u_{\mathrm{s}}}
$$

Problem: $H \nsucc 0$ is indefinite! Solution:

- Check for stability of the local LQ approximation

- Compute $\tilde{H}, \tilde{P} \succ 0$ s.t. $\mathcal{R}_{N}\left(A, B, Q, S, R, P_{N}\right)=\mathcal{R}_{N}\left(A, B, \tilde{Q}, \tilde{S}, \tilde{R}, \tilde{P}_{N}\right)$ 


\section{Look at the Steady State Properties}

Lagrangian of steady state problem

$$
\mathcal{L}=I(x, u)-\lambda^{T}(x-f(x, u))
$$

Lagrangian Hessian:

$$
H=\left[\begin{array}{ll}
Q & S^{T} \\
S & R
\end{array}\right]=\left.\frac{\partial^{2} \mathcal{L}}{\partial(x, u)^{2}}\right|_{x_{\mathrm{s}}, u_{\mathrm{S}}}
$$

Local linearization

$$
A=\left.\frac{\partial f(x, u)}{\partial x}\right|_{x_{\mathrm{s}}, u_{\mathrm{s}}} \quad B=\left.\frac{\partial f(x, u)}{\partial u}\right|_{x_{\mathrm{s}}, u_{\mathrm{s}}}
$$

Problem: $H \nsucc 0$ is indefinite!

Solution:

- Check for stability of the local LQ approximation

- Compute $\tilde{H}, \tilde{P} \succ 0$ s.t. $\mathcal{R}_{N}\left(A, B, Q, S, R, P_{N}\right)=\mathcal{R}_{N}\left(A, B, \tilde{Q}, \tilde{S}, \tilde{R}, \tilde{P}_{N}\right)$

- Formulate a tracking NMPC scheme using $\tilde{H}$ 


\section{Example}

- System dynamics: $\dot{x}=\frac{u}{10}(1-x)-0.4 x$, (discr.: 50 RK4 steps, $\left.\Delta=0.5\right)$ 


\section{Example}

- System dynamics: $\dot{x}=\frac{u}{10}(1-x)-0.4 x$, (discr.: 50 RK4 steps, $\left.\Delta=0.5\right)$

- Stage cost: $I(x, u)=-2 u x-1.5 u+0.1(u-4)^{2}$ 


\section{Example}

- System dynamics: $\dot{x}=\frac{u}{10}(1-x)-0.4 x$, (discr.: 50 RK4 steps, $\left.\Delta=0.5\right)$

- Stage cost: $I(x, u)=-2 u x-1.5 u+0.1(u-4)^{2}$

- Steady state: $\left(x_{\mathrm{s}}, u_{\mathrm{s}}\right)=(0.5,4)$ 


\section{Example}

- System dynamics: $\dot{x}=\frac{u}{10}(1-x)-0.4 x$, (discr.: 50 RK4 steps, $\left.\Delta=0.5\right)$

- Stage cost: $I(x, u)=-2 u x-1.5 u+0.1(u-4)^{2}$

- Steady state: $\left(x_{\mathrm{s}}, u_{\mathrm{s}}\right)=(0.5,4)$

- Approx. EMPC: $I_{\mathrm{tr}}^{0}(x, u)=\left[\begin{array}{l}x-x_{\mathrm{s}} \\ u-u_{\mathrm{s}}\end{array}\right]^{T}\left[\begin{array}{rr}14.227 & 0.825 \\ 0.825 & 0.066\end{array}\right]\left[\begin{array}{l}x-x_{\mathrm{s}} \\ u-u_{\mathrm{s}}\end{array}\right]$ 


\section{Example}

- System dynamics: $\dot{x}=\frac{u}{10}(1-x)-0.4 x$, (discr.: 50 RK4 steps, $\left.\Delta=0.5\right)$

- Stage cost: $I(x, u)=-2 u x-1.5 u+0.1(u-4)^{2}$

- Steady state: $\left(x_{\mathrm{s}}, u_{\mathrm{s}}\right)=(0.5,4)$

- Approx. EMPC: $I_{\mathrm{tr}}^{0}(x, u)=\left[\begin{array}{l}x-x_{\mathrm{s}} \\ u-u_{\mathrm{s}}\end{array}\right]^{T}\left[\begin{array}{rr}14.227 & 0.825 \\ 0.825 & 0.066\end{array}\right]\left[\begin{array}{l}x-x_{\mathrm{s}} \\ u-u_{\mathrm{s}}\end{array}\right]$

- Naive tracking: $l_{\mathrm{tr}}^{1}(x, u)=\left(x-x_{\mathrm{s}}\right)^{2}+\left(u-u_{\mathrm{s}}\right)^{2}$ 


\section{Example}

- System dynamics: $\dot{x}=\frac{u}{10}(1-x)-0.4 x$, (discr.: 50 RK4 steps, $\left.\Delta=0.5\right)$

- Stage cost: $I(x, u)=-2 u x-1.5 u+0.1(u-4)^{2}$

- Steady state: $\left(x_{\mathrm{s}}, u_{\mathrm{s}}\right)=(0.5,4)$

- Approx. EMPC: $I_{\mathrm{tr}}^{0}(x, u)=\left[\begin{array}{l}x-x_{\mathrm{s}} \\ u-u_{\mathrm{s}}\end{array}\right]^{T}\left[\begin{array}{rr}14.227 & 0.825 \\ 0.825 & 0.066\end{array}\right]\left[\begin{array}{l}x-x_{\mathrm{s}} \\ u-u_{\mathrm{s}}\end{array}\right]$

- Naive tracking: $I_{\mathrm{tr}}^{1}(x, u)=\left(x-x_{\mathrm{s}}\right)^{2}+\left(u-u_{\mathrm{s}}\right)^{2}$
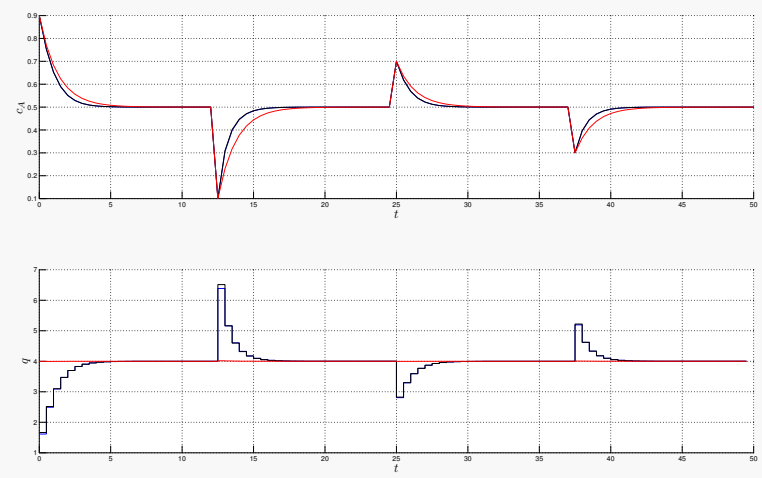
Thank you for your attention! 